\title{
Editorial: Covid-19 Mechanisms on Cardio-Vascular Dysfunction: From Membrane Receptors to Immune Response
}

\author{
Amarylis C. B. A. Wanschel ${ }^{1 *}$, Ana I. S. Moretti ${ }^{2 *}$ and Mireille Ouimet ${ }^{3,4 *}$ \\ ${ }^{1}$ Interdisciplinary Stem Cell Institute, Miller School of Medicine, University of Miami, Miami, FL, United States, ${ }^{2}$ Instituto de \\ Assistência Médica ao Servidor Público Estadual (IAMSPE), Post-Graduation in Health Sciences Program, São Paulo, Brazil, \\ ${ }^{3}$ University of Ottawa Heart Institute, Ottawa, ON, Canada, ${ }^{4}$ Department of Biochemistry, Microbiology and Immunology, \\ Faculty of Medicine, University of Ottawa, Ottawa, ON, Canada
}

Keywords: COVID-19, SARS-CoV-2, endothelium dysfunction, hypertension, obesity, diabetes, cardiovascular disease

\section{Editorial on the Research Topic}

Covid-19 Mechanisms on Cardio-Vascular Dysfunction: From Membrane Receptors to Immune Response

\section{OPEN ACCESS}

Edited and reviewed by: Ichiro Manabe,

Chiba University, Japan

*Correspondence: Amarylis C. B. A. Wanschel awansche/@med.miami.edu Ana I. S. Moretti aismoretti@gmail.com Mireille Ouimet

Mireille.Ouimet@uottawa.ca

Specialty section:

This article was submitted to Cardiovascular Metabolism,

a section of the journal

Frontiers in Cardiovascular Medicine

Received: 27 March 2021 Accepted: 30 March 2021 Published: 27 April 2021

Citation: Wanschel ACBA, Moretti AIS and Ouimet M (2021) Editorial: Covid-19 Mechanisms on Cardio-Vascular Dysfunction: From Membrane Receptors to Immune Response. Front. Cardiovasc. Med. 8:686495.

doi: 10.3389/fcvm.2021.686495
In this special issue of Frontiers in Cardiovascular Medicine, we assembled a collection of hypothesis and theory (1), methods (1), opinion (1), original research articles (1) and reviews (4), within an over-arching theme of "Exploring the COVID-19 mechanisms from membrane receptors to immune response" that uniquely reaffirmed our mission to understand and treat COVID-19. A comprehensive retrospective first takes us through the basic science of pre-existing diseases associated with increased COVID-19 severity, uncovering possible underlying mechanisms potentiating COVID-19 progression.

Coronavirus disease 2019 (COVID-19) has reached a global outbreak, increasing to pandemic proportion, and pre-existing chronic conditions such as cardiovascular disease, hypertension, diabetes, and obesity are major contributors to its morbidity and mortality worldwide (Figure 1). During severe acute respiratory syndrome coronavirus 2 (SARS-CoV-2) infection, enveloped, positive single-stranded RNA virus is endocytosed by epithelial and endothelial cells through the angiotensin-converting enzyme 2 (ACE2) receptor, requiring virus spike-ACE2 interactions and transmembrane serine protease-2 (TMPRSS2) priming (Figure 1). Expression of the ACE2 entry receptor for SARS-CoV-2 in vascular cells is upregulated in individuals with comorbidities, accelerating the course of disease development. Furthermore, direct viral infection of cardiomyocytes is possible due to ACE2 expression in the heart (Figure 1). In contrast, with less functional ACE2, the pediatric population appears to be less vulnerable to COVID-19 infection than adults.

The molecular basis, potential pathways and the role of endothelium in the pathogenesis of cardiac and vascular injuries in COVID-19 were discussed in a new study by Hachim et al. Notably, the common cardiomyocyte protective genes SON, OGT, and RORA were significantly downregulated in cardiovascular comorbidities (acute coronary syndrome, heart failure, venous thromboembolism) compared to controls and in the healthy endothelium of African Americans compared to Caucasians, suggesting that individuals who have a pre-existing genetic propensity for low expression of these genes may be more susceptible to cardiac damage during COVID19. Recent studies have shown that cardiovascular comorbidity plays a role in cardiac injury in COVID-19 patients (Iacobazzi et al.). Furthermore, SARS-CoV-2-induced downregulation of 


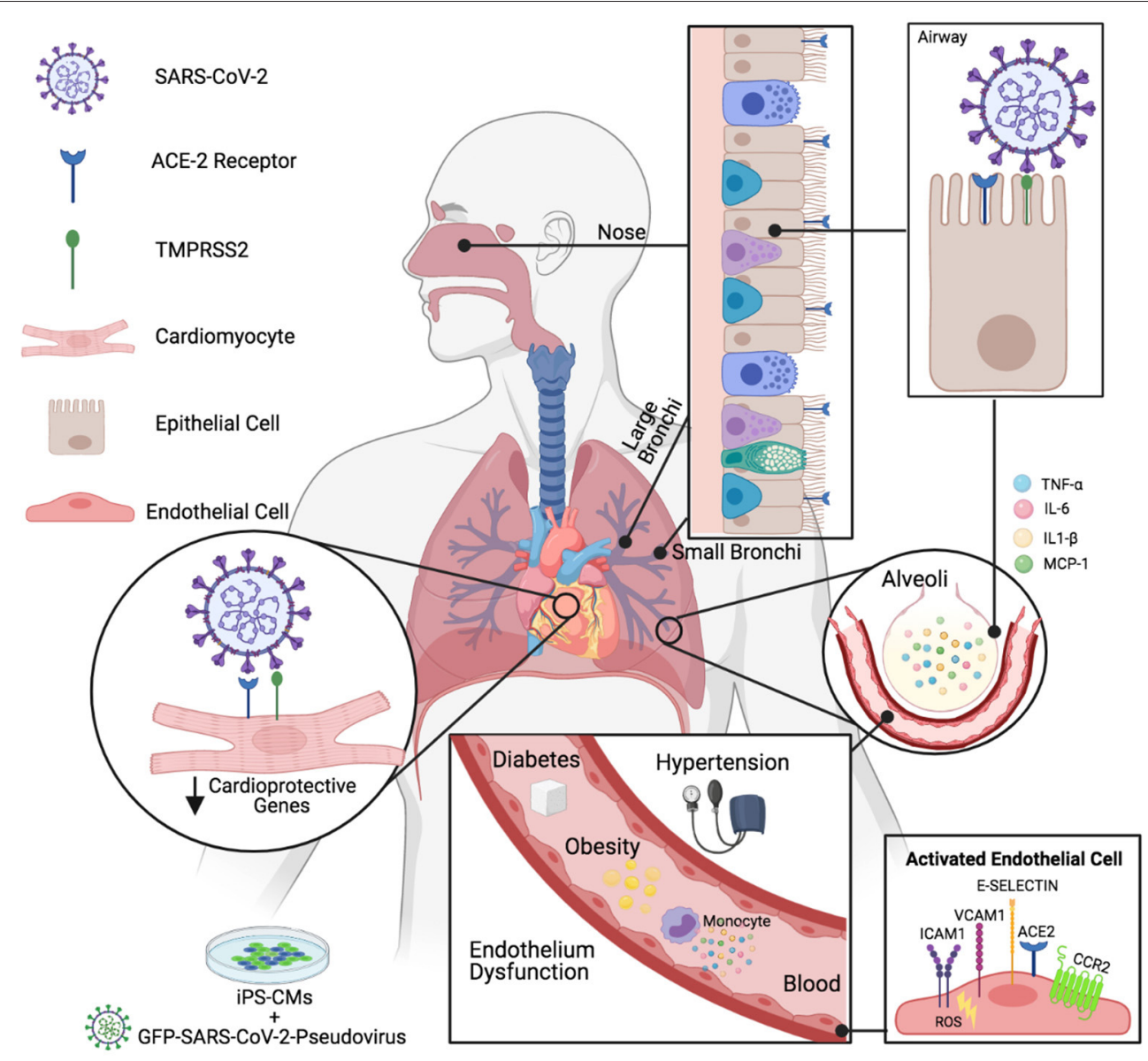

FIGURE 1 | COVID-19 on Comorbidities (created with Biorender.com). Cardiovascular injuries contribute to disease severity during SARS-CoV-2 infection. ACE2, Angiotensin-converting enzyme 2; TMPRSS2, Transmembrane serine protease 2; iPS-CMs, Human inducible pluripotent stem cell-derived cardiomyocytes; GFP-SARS-CoV-2, Green fluorescent protein bearing SARS-CoV-2 pseudovirus; TNF $\alpha$, Tumor necrosis fator alfa; (IL)-6, (IL)-1 13 , Interleukin; MCP-1, Monocyte chemoattractant protein 1; VCAM-1, Vascular cell adhesion molecule-1; ICAM-1, Intercellular adhesion molecule-1; CCR2, Chemokine receptor type 2; ROS, Reactive oxygen species.

another set of cardioprotective genes NDUFA4L2, NDUFB7, MRPS11, and HIKESHI, concomitant with upregulation CHD9, $M T F 2, R O R A, M Y C$, and ETS1 may promote cardiac injury upon SARS-CoV-2 infection (Hachim et al.) (Figure 1).

Vascular dysfunction is the common link between comorbidities and increased severity of COVID-19 infection; however, the factors that trigger endothelium injury differ in every common comorbidity in patients with COVID-19. During diabetes, hyperglycemia is associated with massive circulating and endothelial protein glycosylation, leading to elevated oxidative stress that damages blood vessel linings. The role of endothelial cells in SARS-CoV-2 viral infection was extensively reviewed by Hol Fosse et al., highlighting endothelial cell tropism, detection and response, along with the breakdown of vascular function during systemic hyperinflammatory response in COVID-19, as prominent features of human infection. An opinion article by Sur et al. shed light on the underlying mechanisms by which diabetes might augment the severity of
COVID-19. Hyperglycemia may have a strong association with low intracellular $\mathrm{pH}$ and could potentiate the SARS-CoV-2 infection (Sur et al.). In addition, hyperglycemia promotes the synthesis of inflammatory cytokines, and diabetic patients with COVID-19 are more susceptible to immune cell hyperactivation, or a cytokine storm (Sur et al.).

Type 1 or type 2 diabetic patients treated with angiotensin converting enzyme inhibitors (ACEIs) or angiotensin-receptor blockers (ARBs) exhibit increased ACE2 receptor expression and could therefore be at a higher risk of SARS-CoV-2 infection and severity of COVID-19. However, changes in the expression of ACE2 in diabetes vary across different organs, and the impact of such changes on COVID-19 severity are still under investigation. Other meta-analyses suggested no significant association between the use of ACEIs or ARBs. Roberts et al. also highlight that hyperglycemia impairs host defense, and that blood glucose itself is a key mediator of severe symptoms in diabetic patients infected with SARS-CoV-2. The endothelium 
of diabetic patients is already compromised by hyperglycaemiainduced oxidative stress, driving endothelium activation, and increasing the expression of adhesion molecules, such as vascular cell adhesion molecule 1 (VCAM-1), intercellular adhesion molecule 1 (ICAM-1) and E-Selectin that orchestrate leukocyte recruitment (Figure 1).

Chronic and persistent immune dysregulation in type 1 or type 2 diabetes includes lower rates of phagocytosis by neutrophil and macrophages that is necessary for uptake of infectious antigen, in addition to suppressed chemokine responses and higher levels of pro-inflammatory mediators. Because lower respiratory tract infections occur when the immune response in the upper airways fails to contain the viral spread, the route of entry of SARS-CoV-2 in the lungs and the intimate proximity of the endothelium expressing ACE2 and alveolar epithelial cells together with resident macrophages compound COVID-19 severity in diabetic patients. Lung autopsies of deceased COVID-19 patients demonstrated that neutrophil extracellular traps (NETs), which are networks of extracellular fibers composed of DNA from neutrophils that bind pathogens, were likely to be involved in inflammation-associated lung damage, thrombosis, and fibrosis. To date, generation of NETs is a common feature in diabetes and infection, boosted by COVID-19. In diabetic patients, a potential primer for thrombosis in COVID-19 is increased circulating inflammatory cytokines, along with endothelial dysfunction as a crucial determinant of thrombotic potential through loss of antithrombotic nitric oxide (NO), prostaglandin 2 (PGI2), imbalance of fibrinolytic plasminogen activator inhibitor t-PA/PAI-1, and platelet hyperactivity, all of which are exacerbated by oxidative stress (Sur et al.).

The luminal surface of vascular endothelial cells is covered by a gel-like of interconnected proteins, the glycocalyx that plays a role in vascular permeability, adhesion of leucocytes and platelets, and modulation of inflammatory processes. Plasma-derived albumin is homogeneously distributed onto the endothelial glycocalyx layer (EGL). Interestingly, SARS-CoV-2 virions are transported by albumin, inhibiting free albumin binding to glycocalyx and disrupting normal fluid homeostasis of the microvasculature (Johnson et al.). Therefore, albumin therapy to replace SARS-CoV-2-bound albumin may improve vascular permeability and alleviate some of the COVID-19 symptoms leading to sepsis.
More studies are needed to evaluate the effect of SARS$\mathrm{CoV}-2$ and potential therapies on populations classified as risk groups. Capcha et al. have developed a protocol for in vitro studies of SARS-CoV-2 pseudovirus infection in renal and lung epithelial cells and in human induced pluripotent stem cell-derived cardiomyocytes (iPS-CMs) (Figure 1). Such models will prove useful to further evaluate the potential for therapeutic approaches to control SARS-CoV-2 infection, such as the recently described ultraviolet light that could modulate morbidity and mortality from COVID-19 (Gorman et al.) and cell-based therapy to attenuate cytokine storm (Iacobazzi et al.). Further understanding of the interactions of COVID-19 with the cardiovascular system will help the design of future therapeutic approaches to reduce morbidity in patients with underlying cardiovascular complications.

\section{AUTHOR CONTRIBUTIONS}

The authors equally contributed to the drafting revision of the editorial and approved it for publication.

\section{FUNDING}

This work was supported by funding from the Canadian Institutes for Health Research (PJT-391187 and Canada Research Chair to MO) and the National Sciences and Engineering Research Council of Canada (Discovery Grant to $\mathrm{MO})$.

\section{ACKNOWLEDGMENTS}

We thank all contributing authors and reviewers for their support to the Research Topic.

Conflict of Interest: The authors declare that the research was conducted in the absence of any commercial or financial relationships that could be construed as a potential conflict of interest.

Copyright $\odot 2021$ Wanschel, Moretti and Ouimet. This is an open-access article distributed under the terms of the Creative Commons Attribution License (CC BY). The use, distribution or reproduction in other forums is permitted, provided the original author(s) and the copyright owner(s) are credited and that the original publication in this journal is cited, in accordance with accepted academic practice. No use, distribution or reproduction is permitted which does not comply with these terms. 